\title{
Rapid Spontaneous Remission of a Spontaneous Spinal Chronic Subdural Hematoma in a Child
}

\author{
-Case Report-
}

\author{
Dong-Hyuk PARK, Tai-Hyoung CHO, Jang-Bo LEE, Jung-Yul PARK, \\ Youn-Kwan PARK, Yong-Gu CHUNG, and Jung-Keun SUH \\ Department of Neurosurgery, Korea University, College of Medicine, Seoul, R.O.K.
}

\begin{abstract}
A 13-year-old boy presented with a rare spontaneous spinal chronic subdural hematoma (SCSDH) with no associated trauma or medical problems manifesting as back and bilateral lower extremity pain persisting for 10 days. Neurological examination revealed mild weakness and paresthesia in both lower extremities. Magnetic resonance (MR) imaging performed 1 week after the appearance of symptoms revealed a chronic subdural hematoma at the thoracolumbosacral region. Follow-up MR imaging performed 1 week later showed significant resolution of the hematoma without the need for surgery. The patient was discharged with only conservative management. This case of spontaneous SCSDH with rapid spontaneous remission in a child not associated with coagulopathy indicates that aggressive surgical treatment should be delayed as long as possible in pediatric patients because the spinal structure is still developing.
\end{abstract}

Key words: child, chronic subdural hematoma, spinal subdural hematoma, spontaneous remission

\section{Introduction}

Spinal chronic subdural hematoma (SCSDH) is a rare disease that can occur in patients who have suffered minor trauma, coagulopathy, or anticoagulation therapy. Spontaneous SCSDH is even rarer, and is occasionally associated with chronic intracranial hematoma, ${ }^{1,11,19)}$ superficial hemosiderosis of the brain, ${ }^{15)}$ spinal arachnoiditis, or syringomyelia. ${ }^{18)}$ Nevertheless, the etiology and pathogenesis of spontaneous SCSDH remain unclear because unlike the cerebral subdural space, the spinal subdural space does not contain bridging vessels and is protected by the vertebral column as well as several paravertebral muscles. ${ }^{2,12,14,19)}$ Only five cases of spontaneous SCSDH without specific pathology have been reported. ${ }^{3,4,6,8,17)}$ We report another case of spontaneous SCSDH in a child with no associated trauma or medical problems that resolved spontaneously.

Received August 14, 2007; Accepted January 23,

\section{Case Report}

A 13-year-old boy presented with back and bilateral lower extremity pain persisting for 10 days. The patient had no previous history of trauma or bleeding diathesis. The descending pain had become aggravated. Neurological examination revealed motor strength of grade $4+$ and mild paresthesia in both lower extremities, paraspinal muscular spasm, and marked tenderness of the lumbar spinal processes. Straight leg rising was limited to 15 degrees, but the deep tendon reflexes remained within the normal limits, and there were no pathological reflexes. Magnetic resonance (MR) imaging, performed at a local clinic 1 week after the back pain had begun, showed a longitudinal space-occupying lesion encircling the cauda equina in the spinal canal from $\mathrm{T} 12$ to $\mathrm{S} 1$. The lesion appeared hyperintense on both $\mathrm{T}_{1^{-}}$and $\mathrm{T}_{2^{-}}$ weighted images but without enhancement after infusion of intravenous gadolinium (Fig. 1).

The patient was transferred to the Department of Neurosurgery at the Anam Hospital of Korea University for further evaluation and management 3 days after the initial MR imaging. Routine laboratory analyses, including complete blood counts and coagulation screen, were within the normal limits. 

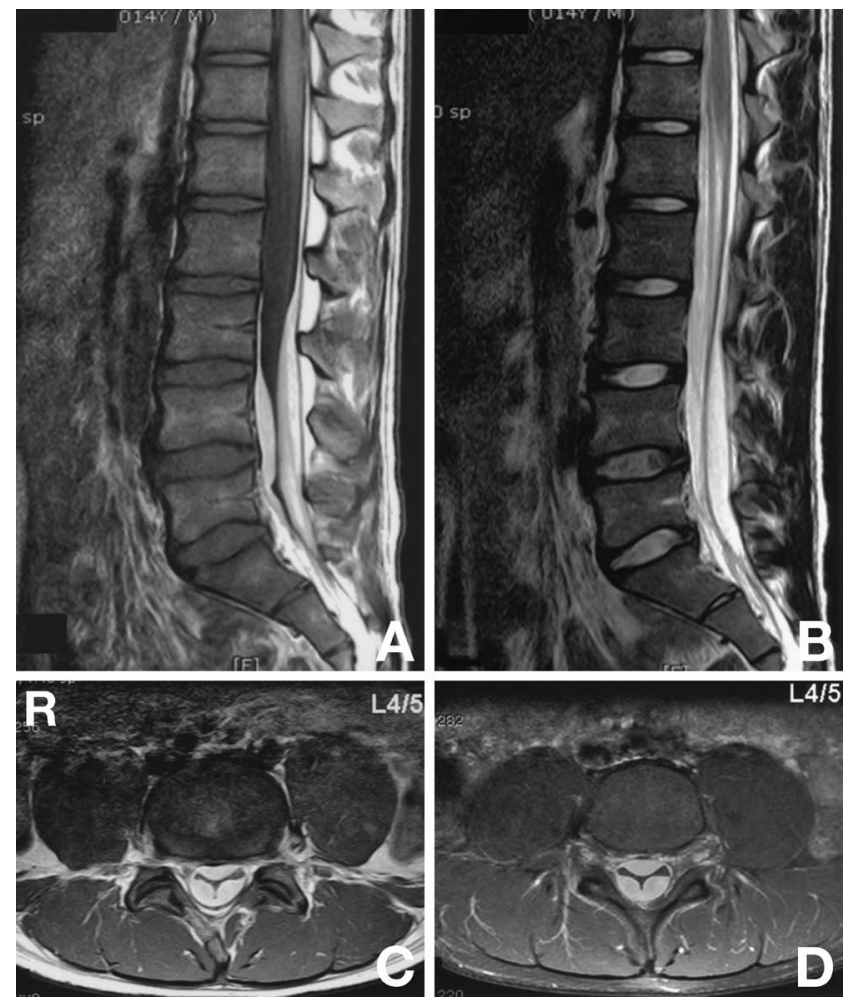

Fig. 1 Sagittal $\mathrm{T}_{1}$-weighted (A) and $\mathrm{T}_{2}$-weighted (B) magnetic resonance images 1 week after the onset demonstrating a hyperintense longitudinal lesion anterior and posterior to the dural sac, extending from T12 within the posterior subdural space and from L3 within the anterior subdural space down to $S 1$, and compressing the cauda equine. Axial $\mathrm{T}_{1}$-weighted images (C) and with gadolinium (D) showing no enhancement of the lesion (L4-5 disc level), with the arachnoid membrane and subarachnoid components surrounded by the subdural hematoma appearing as an inverted "Mercedes star sign."

Emergency surgery was withheld and follow-up MR imaging was performed 3 days after admission to the hospital because of the tolerable neurological signs. Neuroimaging revealed remarkable resolution of the hematoma. His symptoms and the neurological signs had improved progressively. The small residual hematoma extended only from L3 down to L5. However, the lesion remained hyperintense on both $\mathrm{T}_{1^{-}}$and $\mathrm{T}_{2}$-weighted images (Fig. 2). Extensive spinal angiography and bilateral internal iliac angiography did not reveal any evidence of spinal vascular malformations such as arteriovenous malformation or dural arteriovenous fistula.

Conservative management was chosen because
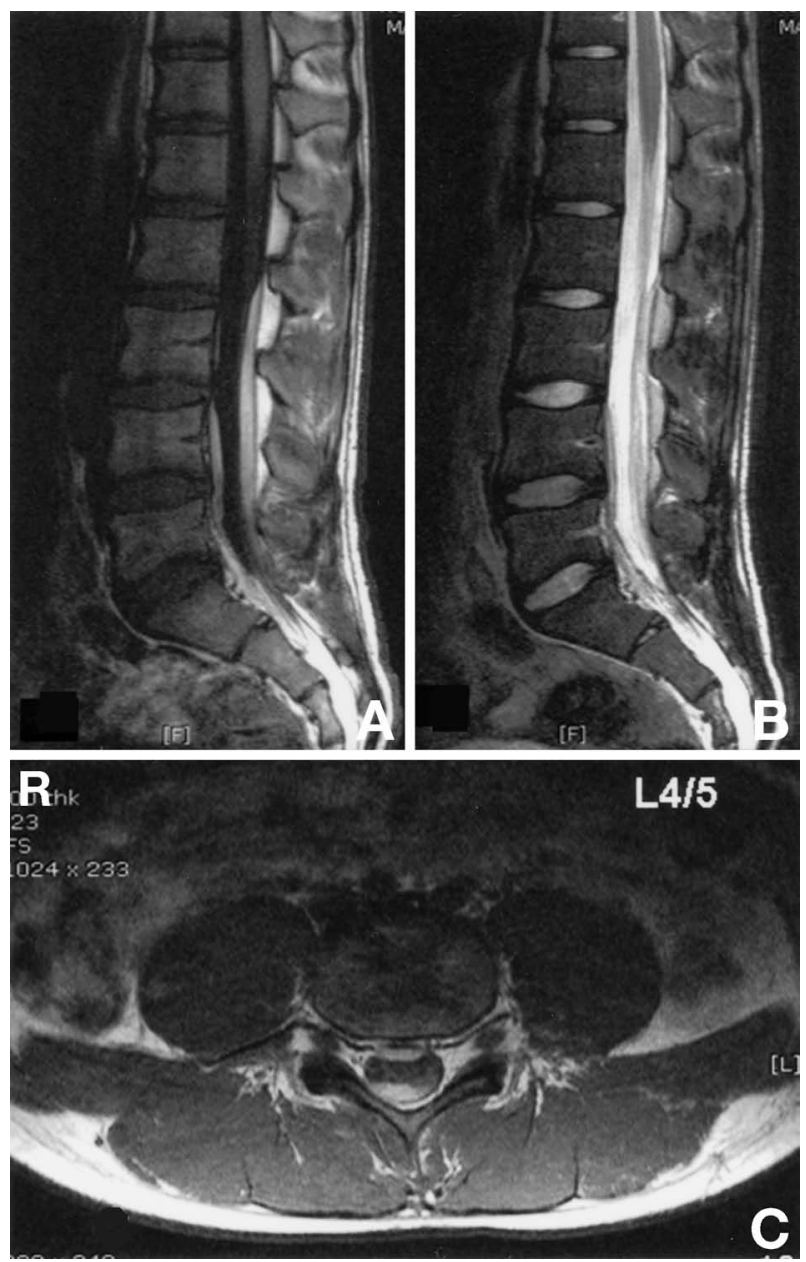

Fig. 2 Sagittal $\mathrm{T}_{1}$-weighted (A) and $\mathrm{T}_{2}$-weighted (B), and axial $\mathrm{T}_{1}$-weighted (C) (L4-5 disc level) magnetic resonance images 1 week later showing significant resolution of the hematoma, with the small residual hematoma extending only from L3 down to L5.

his back pain had recovered rapidly with gradual improvement in the neurological deficits of the bilateral extremities, and the spinal structure was still developing at this young age. He was discharged in almost normal neurological condition 4 weeks after the onset of back pain.

\section{Discussion}

Two hypotheses have been proposed to explain the pathogenesis of SCSDH. First, initial hemorrhage in the subarachnoid space is the primary lesion that eventually dissects into the subdural space, and the subarachnoid hemorrhage is washed out by the cerebrospinal flow through the transitional state, ${ }^{6,7,9,16,17)}$ as supported by observations of the transitional state of combined subarachnoid hem- 
orrhage and subdural hematoma., ${ }^{2,7)}$ Second, spinal subdural hematoma might be related to the redistribution of blood from the intracranial subdural space, and expansion may be secondary to chronic changes, ${ }^{1)}$ supported by a possible association between SCSDH and intracranial chronic subdural hematoma. ${ }^{1,11,19)}$

The mechanism of the spontaneous resolution of SCSDH is also unknown. The direction of the movement of a hematoma might be reversed, namely from the subdural to subarachnoid space. ${ }^{13)}$ The liquid subdural hematoma may reversely penetrate into the subarachnoid space through the broken arachnoid membrane prior to healing, and is then washed out by the cerebrospinal fluid flow. Dissection of subdural hematoma into the subarachnoid space can occur gradually because of the time interval of clot lysis. However, in our case, the cause of the prompt spontaneous resolution is still unclear.

MR imaging recognition of the blood products is the most important sign that distinguishes SCSDH from other extramedullary spinal lesions. Acute subdural hematoma is isointense with the spinal cord on $\mathrm{T}_{1}$-weighted imaging and mixed intensity on $\mathrm{T}_{2^{-}}$ weighted imaging, with peripheral hyperintensity on both $\mathrm{T}_{1^{-}}$and $\mathrm{T}_{2}$-weighted imaging at approximately 4-7 days after bleeding. In the late chronic stage, hyperintensity is typically observed on both $\mathrm{T}_{1^{-}}$and $\mathrm{T}_{2}$-weighted imaging. ${ }^{10,19)}$ Extramedullary tumor or abscess will usually appear with rim or uniform enhancement on $\mathrm{T}_{1}$-weighted imaging, whereas SCSDH shows no contrast enhancement. ${ }^{10)}$ Axial images are essential for differentiating between the epidural and subdural locations of the hematoma. ${ }^{10,19)}$ Spinal epidural hematoma has a more lentiform shape, and occasionally extends into the intervertebral foramina. In contrast, spinal subdural hematoma has a crescent shape and traps the spinal cord or cauda equina. An inverted "Mercedes star sign" may indicate a subdural location in the lumbar spine. ${ }^{10)}$ These findings were also observed in our case (Fig. 1).

Only five previous cases of spontaneous SCSDH without pathology have been reported..$^{3,4,6,8,17)}$ The lumbar ${ }^{4,8)}$ and thoracolumbar ${ }^{3,6)}$ regions were the most common sites for the spontaneous SCSDH. Only one case was a cervical SCSDH. ${ }^{17)}$ All five patients, including one pediatric patient, underwent laminectomy to evacuate the SCSDH. ${ }^{4)}$ Nontraumatic acute spinal subdural hematomas in the pediatric patients are almost always associated with bleeding disorders and poor outcomes. ${ }^{5)}$ The present pediatric case of spontaneous SCSDH in a child was not associated with coagulopathy at the thoracolumbosacral region and showed rapid spontaneous remission.

Emergency surgical decompression is generally the best treatment in patients presenting with acute deterioration and severe neurological deficits such as acute spinal subdural hematoma. Percutaneous drainage might be an option in patients with stable deficit, dorsally located hematoma of moderate extent in the lumbosacral area, and no bleeding diathesis. ${ }^{2)}$ Conservative management might be an option in patients with mild neurological deficits and progressive improvement in the early period, as seen in our case. In particular, aggressive surgical treatment should be delayed as long as possible in pediatric patients because the spinal structure is still developing.

\section{References}

1) Bortolotti C, Wang H, Fraser K, Lanzino G: Subacute spinal subdural hematoma after spontaneous resolution of cranial subdural hematoma: causal relationship or coincidence? Case report. J Neurosurg 100: 372-374, 2004

2) Brandt RA: Chronic spinal subdural haematoma. Surg Neurol 13: 121-123, 1980

3) Calhoun JM, Boop F: Spontaneous spinal subdural hematoma: case report and review of the literature. Neurosurgery 29: 133-134, 1991

4) Chaves CJ, Bianco E, Shibata MK, de Almeida GM: Chronic spinal subdural hematoma. Surg Neurol 40: 435-436, 1993

5) Domenicucci M, Ramieri A, Ciappetta P, Delfini R: Nontraumatic acute spinal subdural hematoma: report of five cases and review of the literature. J Neurosurg 91: 65-73, 1999

6) Harris W: Two cases of spontaneous hematorrhachis or intrameningeal spinal hemorrhage: one cured by laminectomy. Proc R Soc Med 5: 115-122, 1911

7) Kang HS, Chung CK, Kim HJ: Spontaneous spinal subdural hematoma with spontaneous resolution. Spinal Cord 38: 192-196, 2000

8) Khosla VK, Kak VK, Mathuriya SN: Chronic spinal subdural hematomas. Report of two cases. J Neurosurg 63: 636-639, 1985

9) Kirkpatrick D, Goodman SJ: Combined subarachnoid and subdural spinal hematoma following spinal puncture. Surg Neurol 3: 109-111, 1975

10) Kirsch EC, Khangure MS, Holthouse D, McAuliffe $\mathrm{W}$ : Acute spontaneous spinal subdural haematoma: MRI features. Neuroradiology 42: 586-590, 2000

11) Leber KA, Pendl G, Kogler S, Kammerhuber F, Ebner F: Simultaneous spinal and intracranial chronic subdural hematoma. Case illustration. J Neurosurg 87: 644, 1997

12) Mattle H, Sieb JP, Rohner M, Mumenthaler M: Nontraumatic spinal epidural and subdural hematomas. Neurology 37: 1351-1356, 1987

13) Mavroudakis N: Spontaneous spinal subdural hema- 
toma: case report and review of the literature. Neurosurgery 30: 652, 1992

14) Nicholas DS, Weller RO: The fine anatomy of the human spinal meninges. A light and scanning electron microscopy study. J Neurosurg 69: 276-282, 1988

15) Niederhofer H, Huber M: Chronic spinal hematoma as a possible reason for cognitive impairment. Pediatr Neurol 28: 223-224, 2003

16) Rader JP: Chronic subdural hematoma of the spinal cord: report of a case. $N$ Engl J Med 253: 374-376, 1955

17) Russell NA, Benoit BG: Spinal subdural hematoma. A review. Surg Neurol 20: 133-137, 1983

18) Siddiqi F, Hammond R, Lee D, Duggal N: Spontaneous chronic spinal subdural hematoma associated with spinal arachnoiditis and syringomyelia. J Clin Neurosci 12: 949-953, 2005

19) Tillich M, Kammerhuber F, Reittner P, Leber KA, Szolar DH: Chronic spinal subdural haematoma associated with intracranial subdural haematoma: CT and MRI. Neuroradiology 41: 137-139, 1999

Address reprint requests to: Tai-Hyoung Cho, M.D., Department of Neurosurgery, Korea University Anam Hospital, Korea University, College of Medicine, 126-1, 5 ga, Anam-dong, Sungbuk-gu, Seoul, 136-705, R.O.K.

e-mail: choth2@unitel.co.kr 